\title{
The comparative pathogenicity of strains of eight serovars and untypable strains of Mannheimia haemolytica in experimental pneumonia of sheep
}

\author{
Moses O. OduGBO ${ }^{\mathrm{a} *}$, Lillian E. OdAMA ${ }^{\mathrm{b}}$, Jarlath U. $\mathrm{UMOH}^{\mathrm{c}}$, \\ Lami H. LOMBIN ${ }^{\mathrm{a}}$ \\ a National Veterinary Research Institute, Vom, Plateau State, Nigeria \\ b National Institute for Pharmaceutical Research and Development, Abuja, Nigeria \\ c Department of Veterinary Public Health and Preventive Medicine, Ahmadu Bello University, \\ Zaria, Nigeria
}

(Received 20 November 2003; accepted 17 May 2004)

\begin{abstract}
The experimental induction of pneumonic pasteurellosis in groups of conventionally reared lambs by 8 serovars (A1, A2, A6, A7, A8, A9, T10, and A11) and untypable (UT) strains of Mannheimia haemolytica $(\mathrm{Mh})$ were examined and compared. The groups of lambs were inoculated intratracheally with $1.4 \times 10^{8} \pm 0.6 \times 10^{8}$ (mean $\pm \mathrm{SD}$ ) colony-forming units of the Mh serovars or UT isolates in the 6-hour log phase of growth. The variables measured as indicators of disease severity were clinical score, percentage lung consolidation and microbiological re-isolation. The clinical parameters for each group were computed daily for 6 days post infection and the lambs which died were necropsied while the remaining lambs were killed on day 7 pi and the extent of lung consolidation was measured. Clinically, the mean scores for the M. haemolytica serovars were A1 (6.1), A2 (18.8), A6 (0.5), A7 (17.4) and A9 (8.5). The mean percent lung lesion scores for $M$. haemolytica serovars were A1 (12.5), A2 (66.3), A6 (5.0), A7 (51.3), A9 (33.8) and A11 (2.5). The percent mean pneumonic lung lesions recorded for groups inoculated with A2, A7 and A9 were significantly $(P<0.05)$ higher than the extent of lung lesions in the other groups. A statistically significant correlation was observed between clinical scores and the severity of the lung lesions $(r=$ $0.96, P<0.01)$. High titres of $M$. haemolytica were recovered from lung lesions, with 10 to100 times the number of organisms inoculated being present in the lung lesions of lambs inoculated with serovars A2 and A7. These data indicate that although M. haemolytica serovars A1, A2, A6, A7, A9 and A11 are important primary lung pathogens of lambs, serovars A2, A7, and A9 are to be regarded as highly virulent strains that have a greater predilection than the other serovars for causing pneumonia in lambs.
\end{abstract}

sheep / pneumonia / Mannheimia haemolytica / comparative / pathogenicity

\section{INTRODUCTION}

Mannheimia (Pasteurella) haemolytica has long been associated with respiratory disease in sheep with climatic and animal management factors being involved in the pathogenesis of the disease $[1,4,13]$. The multiplicity of serovars capable of causing pneumonia and the high rate of nasal carriage in apparently healthy sheep are two of

\footnotetext{
* Corresponding author: odugm@yahoo.com
} 
the major factors, which make pasteurellosis in sheep different from that in other animals. M. haemolytica is a heterogeneous bacterial pathogen. The heterogeneity of this bacterium is evident from the fact that historically, there have been two biotypes of $M$. haemolytica recognized - biotype A consisting of isolates that ferment L-arabinose and biotype $\mathrm{T}$ consisting of isolates that ferment trehalose [23, 24]; together they are represented by 1-17 M. haemolytica serovars $[13,27]$. The 17 recognized serovars of the former M. haemolytica complex have recently been shown to represent three genetically distinct species. Serovars 3, 4, 10 and 15 represent Pasteurella trehalosi [25], serovar 11 represents Mannheimia glucosida while the reference strain for the other serovars belongs to Mannheimia haemolytica (serovar 17 has not been investigated) [2]. In addition to isolates that express these serovars, approximately $10 \%$ of disease isolates recovered from sheep are untypable (UT) [13]. The UT isolates may represent either capsule-deficient strains or distinct species $[6,19]$. Furthermore, extensive epidemiological studies of M. haemolytica strains using molecular typing techniques have shown some significant level of heterogeneity even within strains of the same serovar isolated from various hosts and also from different geographical areas $[7,8,16]$.

There is a wide variation in the prevalence of individual M. haemolytica serovars isolated from the lungs of sheep throughout the world. In Kenya for instance, 15 different serovars of $M$. haemolytica plus several UT strains have been isolated from sheep [18]. In the Nigerian context there is evidence that $M$. haemolytica is a routine isolate from pneumonic ovine and caprine lungs with the identification of at least 8 serovars and UT strains [20,21]. The development of vaccines against ovine pneumonic pasteurellosis will depend on the variety of strain types involved in disease and on the specificity of the bacterium for the host species. Two previous comparative pathogenicity studies separately conducted in Europe [15] and North
America [12] attempted to determine differences between serovars, which might be related to virulence. Aside from these studies, which used presumably, single representative strains and a limited range of $M$. haemolytica serovars, and reported about a decade and a half ago, contemporary studies are lacking. From the foregoing information, it is evident that an account of the relative pathogenicity of a wider spectrum of $M$. haemolytica serovars or strains, obtained from a tropical geographic location would be desirable. In this study, we compared the extent of pneumonic pasteurellosis induced in conventionally reared lambs using multiple strains of eight serovars and UT strains of M. haemolytica isolated from pneumonic lungs of sheep in Nigeria.

\section{MATERIALS AND METHODS}

\subsection{Experimental animals}

Six to seven-month-old conventionally reared lambs free from clinical signs of pneumonia were purchased from private sources. On arrival the animals were treated for internal parasites (Ivomec/Ivermectin, Merck \& Co Inc., Rahway, NJ, USA) and coccidia (Amprolium, Merck \& Co Inc., Rahway, NJ, USA) and vaccinated with a heterologous tissue culture rinderpest vaccine (TCRV) as prophylaxis against the Peste des petits ruminants (PPR) virus four weeks prior to the study. The animals were conditioned to their new environment for four weeks during which nasal swabs were collected three times a week to ensure that the lambs were free of $M$. haemolytica prior to the start of the experiment. In pre-inoculation sera, antibody levels to the M. haemolytica inoculum, as determined by indirect haemagglutination [11], ranged from 1:4 to 1:8 in four lambs and were negative for the other lambs. Vital signs (rectal temperature, pulse and respiratory rates - TPR) were monitored daily to ensure that the lambs remained afebrile and to establish a clinical baseline. The lambs had ad libitum access to fresh 
native grass/hay, supplemented with a grain ration and fresh water throughout the study.

\subsection{Inoculum}

A total of 8 different serovars and UT isolates of M. haemolytica were used. The serovars were A1, A2, A6, A7, A8, A9, T10, A11, and untypable (UT) strains, which are ovine strains from pneumonic lungs of sheep in Nigeria [20]. The isolates were identified by colony morphology, Gram's stain, biochemical reactions [10] and by the use of specific serotyping antisera [11]. In order to allow possible variation in the virulence of isolates of the $M$. haemolytica serovars, the inoculum contained five different isolates of the same serovar (with the exception of P. trehalosi T10 which had only one isolate), which were originally derived from pneumonic lungs of sheep. Thus the probability of not including a virulent isolate was made significantly low by using a cocktail of strains of the same serovar. The organisms were grown on blood agar (Oxoid) and after incubation at $37^{\circ} \mathrm{C}$ for $24 \mathrm{~h}$, a discrete colony of each of the strains were suspended in sterile $20 \mathrm{~mL}$ nutrient broth and incubated at $37^{\circ} \mathrm{C}$ for $6 \mathrm{~h}$ and all five isolates were pooled and the viability and approximate number of the $M$. haemolytica serovar per dose were determined by diluting the mixed broth culture and by a quantitative plate count. The inoculum in each lamb was $4 \times 10^{8} \pm 0.6 \times 10^{8}$ colony-forming units (CFU) of the M. haemolytica serovar in $7 \mathrm{~mL}$ sterile nutrient broth, as used by other investigators [9].

\subsection{Experimental design}

Forty conventionally reared and clinically healthy lambs were allocated to ten groups of four lambs. The lambs were infected intratracheally with $7 \mathrm{~mL}$ of the inoculum. Groups 1 to 9 were inoculated respectively with ovine strains of $M$. haemolytica (Mh) serovars A1, A2, A6, A7, A8, A9, T10, A11, and UT while group 10 served as the sham-inoculated control. The control lambs were inoculated intratracheally with $7 \mathrm{~mL}$ of sterile nutrient broth. Each group of lambs were housed separately in a roofed block building with cross ventilation, in an environmental temperature of $25 \pm 1^{\circ} \mathrm{C}$ and a relative humidity of $50 \%$. They were maintained ad libitum on fresh grass or hay, feed concentrate and fresh water. Clinical scores were computed daily for six days after inoculation and the lambs that died were necropsied. The remaining lambs were euthanized on day 7 post inoculation (pi), by intravenous injection of pentobarbitone sodium. Animal care and manipulations were conducted in accordance with the Consortium Guide for the care of experimental animals, and their use for scientific purpose [3].

A post inoculation assessment of the lambs was carried out based on three components namely: clinical parameters, a measurement of lung lesions at necropsy and microbiological re-isolation. In order to compare the responses of the lambs in the different groups, a scoring system was adopted according to a modified method of Gilmour et al. [14]. This allowed for the comparative studies of the infective rates of the serovars and UT strains.

\subsection{Clinical assessment}

The lambs in each group were examined for signs of clinical illness for a period of six days after inoculation with the M. haemolytica isolates. To avoid any subjective influence the experiment was run as a blind trial in which the identities of the groups were unknown to the clinician and a score for the degree of illness or presence or absence of the basic clinical signs were awarded daily to each lamb. The features of illness which were looked for were the following: pyrexia $\left(\right.$ rectal temperature exceeding $\left.40^{\circ} \mathrm{C}\right)=1.0$; abnormal respiration $($ dyspnoea $)=1.0$; weakness or lethargy $=1.0$; cough $=0.5$; nasal discharge $=0.5$; anorexia (hay/grass only $=0.5$, or hay/grass and grains $=1.0$ ); recumbency $=1.0$. Thus on any day a lamb could score between 0 and six points. All lambs that died prematurely were given a maximum 
score of six points for each day remaining in the experiment. A cumulative score was obtained for each lamb, before the average daily score for each lamb and group was calculated.

\subsection{Necropsy procedure}

A veterinarian unaware of the group to which the lamb belonged examined pathologically the lambs which died or which had been killed by pentobarbitone sodium. Since $M$. haemolytica generally has a tropism for respiratory tissue, necropsy examination was concentrated primarily on the respiratory tissues. The lungs were examined grossly and the extent of involvement was determined by visual observation, palpation and measurement of the lesion. The proportion of the lung surface area that showed consolidation was measured (Length $\times$ Width $\times$ Thickness) with calipers. The extent of pneumonia was evaluated on the basis of the volume of the consolidated lung tissue expressed as a percent of the total volume of the lungs. Representative blocks of lung tissue were selected from each lamb, fixed in $10 \%$ formol saline, dehydrated in alcohol and embedded in paraffin wax. Sections cut at 5 to $6 \mu \mathrm{m}$ were stained by haematoxylin and eosin and were examined with light microscopy.

\subsection{M. haemolytica re-isolation}

During the period of clinical observation, bilateral nasal swabs were taken from the lambs on days 1 to 6 pi for bacterial culture. Viable counts from $10 \%(\mathrm{w} / \mathrm{v})$ lung tissue suspensions were made on blood agar. Samples of tissues were collected from the cranial, diaphragmatic and caudal lobes and cultured for bacteria, and viable counts of M. haemolytica were made from "lesion" and "non-lesion" pools. Bacterial cultures were performed in an attempt to re-isolate the infective inoculum and to determine if other bacteria were present. Representative colonies were identified by standard bacteriological [10] and serological [11] methods.

\subsection{Statistics}

The scores for clinical signs, macroscopic lesions, and re-isolation of inoculum from lung tissues are presented as mean values \pm standard error of the mean (SEM). Analysis of variance was employed to evaluate overall differences amongst the means or groups. Multiple comparisons were done using the Fisher least significant difference (LSD) procedure [26]. Significance estimates for correlations between clinical score and lesion score were from Fisher's Exact F-test [26].

\section{RESULTS}

\subsection{Clinical observations}

Table I summarizes the mean (SEM) clinical scores for the different treatment groups. Overall, 15 of the 40 experimentally inoculated lambs (controls inclusive) exhibited clinical signs of respiratory tract infection. The highest mean clinical score was recorded for the group of lambs inoculated with $M$. haemolytica A2 (18.8 \pm 8.4$)$, which was significantly $(P<0.05)$ higher than only the mean clinical score of the group inoculated with A6 $(0.5 \pm 0.3)$. The mean clinical scores in the groups of lambs inoculated with either M. haemolytica A1 (6.1 \pm 4.7$)$, A7 $(17.4 \pm 8.2)$ or A9 $(8.5 \pm 4.4)$ were less severe than in the A2-inoculated group of lambs, although the differences were not significant. No clinical scores were observed in the groups of lambs inoculated with A8, T10, A11 serovars, UT strains and the control group.

\subsection{Pathological observations}

A total of 17 of the 40 (control lambs inclusive) inoculated lambs developed various degrees of lung lesions at necropsy. Three of the four lambs in the M. haemolytica serovar A1-inoculated lambs had lesions typical of pneumonic pasteurellosis, which affected from 10 to $25 \%$ of the lung volume, 
Table I. Clinical scores and extent of lung lesions in groups of conventional lambs (4 animals per group) after infection with some serovars of M. haemolytica (Mh).

\begin{tabular}{cccccc}
\hline Group No. & Inoculum & No. of deaths & Clinical score* & $\begin{array}{c}\text { No. of lambs with } \\
\text { lung lesions }\end{array}$ & Lung lesions* $(\%)$ \\
\hline 1 & Mh A1 & 0 & $6.1 \pm 4.7^{\mathrm{a}, \mathrm{c}}$ & 2 & $12.5 \pm 7.2^{\mathrm{d}}$ \\
2 & Mh A2 & 2 & $18.8 \pm 8.4^{\mathrm{a}}$ & 4 & $66.3 \pm 19.1^{\mathrm{e}}$ \\
3 & Mh A6 & 0 & $0.5 \pm 0.3^{\mathrm{b}, \mathrm{c}}$ & 2 & $5.0 \pm 2.9^{\mathrm{d}}$ \\
4 & Mh A7 & 2 & $17.4 \pm 8.2^{\mathrm{a}, \mathrm{c}}$ & 4 & $51.3 \pm 18.4^{\mathrm{e}}$ \\
5 & Mh A8 & 0 & 0 & 0 & 0 \\
6 & Mh A9 & 0 & $8.5 \pm 4.4^{\mathrm{a}, \mathrm{c}}$ & 4 & $33.8 \pm 9.9^{\mathrm{e}}$ \\
7 & Mh T10 & 0 & 0 & 0 & 0 \\
8 & Mh A11 & 0 & 0 & 1 & $2.5 \pm 2.5^{\mathrm{d}}$ \\
9 & Mh UT & 0 & 0 & 0 & 0 \\
10 & Nutrient broth & 0 & 0 & 0 & 0 \\
\hline
\end{tabular}

* Data expressed as mean \pm SEM (standard error of mean).

a,b,c,d,e Values in each column with different superscripts differ significantly $(P<0.05)$.

The data expressed in this table are the original data but transformed $\left(\log _{\mathrm{e}}\right)$ data were used for the statistical analysis due to heterologous variances (when transforming these data, the zero values were replaced by a number of 0.001 ).

and the fourth lamb showed no lesion. Two of the four lambs in the M. haemolytica A2inoculated lambs had pneumonic lesions that affected more than $75 \%$ of the lung volume and two of the lambs had lesions involving 25 to $75 \%$ of the lung volume. Two of the four lambs in the M. haemolytica A6-inoculated lambs had focal lung lesions of $10 \%$ but no lesions were observed in the other two. At necropsy, two of the four lambs inoculated with $M$. haemolytica A7 showed pneumonic lesions that affected more than $75 \%$ of the lung volume and two of the other lambs had lesions, which affected 25 to $50 \%$ of the lung volume. All four lambs inoculated with M. haemolytica A9 had a pneumonic lesions that affected from 10 to $50 \%$ of the lung volume. Only one of the four M. glucosida A11-inoculated lambs showed a pneumonic lesion that affected $10 \%$ of the lung volume. No lesions were observed in the lungs of the groups of lambs inoculated with M. haemolytica A8, P. trehalosi T10, UT serovars and the lambs of the control group.

Table I summarizes the mean (SEM) percentage of pneumonic lung lesions recorded for each treatment group. The average extent of the lung lesions in the groups inoculated with A2, A7, and A9 were significantly $(P<$ $0.05)$ more than those in the other groups. The differences between the average extent of lung lesions of the groups inoculated with A2, A7 and A9, and the differences between the average extent of lung lesions of the groups inoculated with A1, A6 and A11 were not significant.

\subsection{Correlation between clinical scores and lung lesions}

There was a strong and significant relationship between clinical scores and the extent of lung lesions, and the overall correlation was significant $(r=0.96, P<0.01)$.

\subsection{Microbiological re-isolation}

No M. haemolytica was isolated from nasal swab specimens of any of the experimental lambs prior to challenge exposure with the live M. haemolytica serovars or UT strains. The number of $M$. haemolytica serovar isolated per treatment group from the 
Table II. Number of M. haemolytica (Mh) isolates recovered from the nasal mucosa, at necropsy from the lungs of lambs (4 animals per group) and mean $M$. haemolytica serovar titre recovered from the lungs.

\begin{tabular}{ccccc}
\hline $\begin{array}{c}\text { Group } \\
\text { No. }\end{array}$ & Inoculum & $\begin{array}{c}\text { No. of nasal } \\
\text { mucosa }\end{array}$ & $\begin{array}{c}\text { No. of } \\
\text { lungs }\end{array}$ & $\begin{array}{c}\text { (CFU/g) } \\
\text { Log }_{10^{*}}\end{array}$ \\
\hline 1 & Mh A1 & 2 & 2 & $4.9 \pm 2.2^{\mathrm{b}, \mathrm{c}}$ \\
2 & Mh A2 & 4 & 4 & $10.0 \pm 0.5^{\mathrm{a}}$ \\
3 & Mh A6 & 0 & 1 & $1.4 \pm 1.5^{\mathrm{c}}$ \\
4 & Mh A7 & 3 & 3 & $9.2 \pm 0.3^{\mathrm{a}}$ \\
5 & Mh A8 & 0 & 0 & 0 \\
6 & Mh A9 & 3 & 3 & $6.7 \pm 2.1^{\mathrm{b}}$ \\
7 & Mh T10 & 0 & 0 & 0 \\
8 & Mh A11 & 0 & 0 & 0 \\
9 & Mh UT & 0 & 0 & 0 \\
10 & Control & 0 & 0 & 0
\end{tabular}

* Mh average titre $\left(\log _{10}\right)$ was calculated from $10 \mathrm{~g}$ $(10 \% \mathrm{w} / \mathrm{v})$ lung tissue taken from "lesion" and "non-lesion" pools blended in $90 \mathrm{~mL}$ sterile nutrient broth (diluent) and serially diluted. For bacterial count data 0 represents no bacteria.

a,b,c Values in each column with different superscripts differ significantly $(P<0.05)$.

nasal and lung locations and the mean bacterial numbers recovered from the lungs following challenge are summarized in Table II. Mean bacterial numbers (cfu/g) of lungs in the animals inoculated with A2 $\left(10^{10.0 \pm 0.5}\right)$, and A7 $\left(10^{9.2 \pm 0.3}\right)$ were significantly $(P<$ $0.05)$ higher than those in the other groups.

\section{DISCUSSION}

The illness and lesions induced in the lambs in this study were in all respects identical with the natural disease, varying only in intensity among groups [1, 13]. Clinically, the lambs infected with $M$. haemolytica serovars A2 and A7 appeared to have suffered the most severe illness, resulting in each case in the death of 2/4 lambs. The lambs infected with serovars A1 and A9 also exhibited severe clinical signs but no death. The groups that received A2, A7 and A9 respectively were distinct in the sense that a good proportion of the lambs exhibited the prominent and serious symptom of dyspnoea in addition to the other constitutional symptoms, making them the most virulent serovars in this study. The dyspnoeic lambs in the groups infected with A2 and A7 eventually died while those in the A9 infected group apparently recovered from the dyspnoeic stage to survive the period of the experiment. Some workers observed that most sheep that reach the dyspnoeic stage of pneumonic pasteurellosis usually die if untreated, or if they survive, they develop into unthrifty chronic respiratory cripples or "lungers" [13].

Pathologically, the fatal lambs in groups that were challenged exposed to M. haemolytica serovars A2 and A7 recorded the severest lung consolidation (mostly over 50\% lung consolidation) with complications of adhesions and pleurisy that were Mannheimia-related. Although there was no fatal case, the extent of lung lesions in serovar A9-infected lambs was comparable to but did not exceed that produced by A2 or A7. The inference from this is that serovars A2, A7 and A9 may have a greater tropism than the other serovars for causing lung injury in lambs. Lambs infected with serovars A1, A6 and A11 generally showed less than 25\% lung consolidation at necropsy. Statistically, a strong correlation between the clinical signs or scores and the extent of lung lesions was observed $(r=0.96, P<0.01)$, which gave the assumption that the appearance of the clinical signs coincided with the pathological progression of the disease; and that the more signs or symptoms present the more severe the disease, which agrees with a similar correlation study by Reeve-Johnson [22].

Microbiologically, the majority of the lambs that showed clinical signs of respiratory disease were positive for the infective serovar from the nasal discharges in this study. It was apparent that in the fatal lambs, 
a high number of organisms were present in the lesions - between 10 to 100 times the number of organisms inoculated. In the nonfatal lambs necropsied on day $7 \mathrm{pi}$, the number of organisms associated with the lesions were less than those inoculated. M. haemolytica was successfully re-isolated from all lung samples with more than $25 \%$ lesions but not always from lungs with less than $25 \%$ lesions. M. haemolytica was, however, not isolated from lungs with without lesions. The implication of this is that lambs with more than $25 \%$ lung lesions are likely to succumb to the disease, whereas lambs with less than $25 \%$ are likely to recover from the disease.

In this study, the low antibody titres to $M$. haemolytica in some of the lambs prior to challenge infection, may indicate either prior exposure to the organism or response to cross-reactive antigens in other species of bacteria since IHA antibodies to M. haemolytica have also been found in the sera of specific pathogen-free lambs which had no experience of $M$. haemolytica [21]. How low levels of antibody may have affected the outcome of this study is unclear, although it has been shown that calves vaccinated with $M$. haemolytica bacterins may have more severe disease than non-vaccinates [17]. Interestingly, one of the lambs with a marked lung consolidation (in the A9-infected group) and one without overt lung lesion (in the A6-infected group) in this study both had the highest pre-inoculation antibody titre $(1: 8)$ to their respective antigens. It is thus difficult to draw any correlation between these low antibody levels and the degree of pneumonia induced.

In previous comparative studies aimed at furthering our understanding of the virulence and pathogenesis of single "representative" strains of different serovars of $M$. haemolytica, Gilmour et al. [15] were unable to demonstrate differences in virulence among isolates of serovars A1, A2, A7, and A9 in sheep. Similarly, Gentry et al. [12] were unable to identify major differences in pathogenicity between 6 isolates of $M$. haemolytica, namely serovars A1, A2, A5, A6, and A9, together with an untypable isolate in calves. Since evidence from recent studies have shown that randomly chosen isolates are not necessarily representative of their respective serovars [8], we employed as the inoculum, a mixture of isolates of the same serovar in contrast to the single "representative" strain of each serovar by previous workers. The results of our study showed that the serovars A1, A2, A6, A7 and $A 9$, in agreement with the results of previous workers, lead to the production of pneumonia in lambs. However, unlike the previous studies, differences were recorded in the degree and extent of the induction of disease among these serovars, with A2 and A7 showing the highest disease scores followed by serovars A9, A1, A6 and A11 in descending order of pathogenicity in lambs.

The findings resulting from this study have an important implication for vaccine development. An effective vaccine should offer protection in the host species against all serovars associated with the disease. The conventional approach has been to incorporate all of the representative strains of the common serovars prevalent in an area as a bacterin cocktail [5]. The inclusion of several serovars in the bacterin may, however, lead to complex formulation, which imposes a very heavy antigenic load on vaccinated animals. To reduce the overall quantity of antigens required in a bacterin, it is desirable to determine the relative pathogenicity of the prevailing serovars in the area, with a view to formulating a vaccine cocktail from the few highly pathogenic serovars or strains. In this study, serovars A2, A7 and A9 proved to be the most important pathogens in the experimental lambs and field reports also agree [13].

Our results lend support to earlier studies [13], which indicate that serovars A1, A2, A6, A7 and A9 are the most resilient to efforts for the effective control of pneumonic pasteurellosis in lambs with serovars A2, A7 and A9 showing the most significant virulence in lambs. 


\section{ACKNOWLEDGMENTS}

The National Veterinary Research Institute, Vom, under the National Agricultural Research Programme (NARP) - World Bank assisted project, supported the study. The authors are grateful to Drs S.O. Ogunjumo, D. Shamaki, A.U. Turaki and K. Yahaya for their contributions. We thank Dr G. Dogo for statistical analysis of the data and Dr W. Donachie, Moredun Research Institute, Edinburgh for the specific $M$. haemolytica antisera.

\section{REFERENCES}

[1] Alley M.R., Pneumonia in sheep, Vet. Annu. 31 (1991) 51-58.

[2] Angen Ø., Mutter R., Caugant D.A., Olsen J.E., Bisgaard M., Taxonomic relationships of the [Pasteurella] haemolytica complex as evaluated by DNA-DNA hybridizations and $16 \mathrm{~S}$ rRNA sequencing with the proposal of Mannheimia haemolytica gen. nov. comb. nov., Mannheimia granulomatis comb. nov., Mannheimia glucosida sp. nov. and Mannheimia varigena sp. nov., Int. J. Syst. Bacteriol. 49 (1999) 67-89.

[3] Anonymous, Guide for the care and use of agricultural animals in agricultural research and teaching. Consortium for developing a guide for the care and use of agricultural animals in agricultural research and teaching. Champaign, Ill: Association Headquarters, National Academy Press, Washington D.C., 1988, pp. 1-74.

[4] Brogden A.K., Lehmkuhl H.D., Cutlip R.C., Pasteurella haemolytica complicated respiratory infections in sheep and goats, Vet. Res. 29 (1998) 233-254.

[5] Chandrasekeran S., Kamal H., Zamri-Saad M.Y., Yeap P.C., Evaluation of combined Pasteurella vaccines in control of sheep pneumonia, Br. Vet. J. 147 (1991) 437-443.

[6] Davies R.L., Paster B.J., Dewhirst F.E., Phylogenetic relationships and diversity within the Pasteurella haemolytica complex based on 16S rRNA sequence comparison and outer membrane protein and lipopolysaccharide analysis, Int. J. Syst. Bacteriol. 46 (1996) 736-744.

[7] Davies R.L., Arkinsaw S., Selander R.K., Genetic relationships among Pasteurella trehalosi isolates based on multilocus enzyme electrophoresis, Microbiology 143 (1997) 28412849.

[8] Davies R.L., Arkinsaw S., Selander R.K., Evolutionary genetics of Pasteurella haemolytica isolates recovered from cattle and sheep, Infect. Immun. 65 (1997) 3585-3593.

[9] Debey B.M., Cutlip R.C., Brogden K.A., Frank G.H., A comparison of intratracheal, intravenous and intratonsillar routes of inoculation of goats with Pasteurella haemolytica, Vet. Res. Commun. 16 (1992) 247-251.

[10] Frank G.H., Serotypes of Pasteurella haemolytica in sheep in Midwestern United States, J. Am. Vet. Res. 43 (1982) 2035-2037.

[11] Fraser J., Donachie W., Quirie M., Gilmour N.J.L., Rapid indirect haemagglutination test for serotyping Pasteurella haemolytica, J. Clin. Microbiol. 18 (1983) 206-207.

[12] Gentry M.J., Confer A.W., Holland S.G., Comparison of the toxic and antigenic properties of single bovine isolates of Pasteurella haemolytica representing five serotypes and an untypable strain, Vet. Microbiol. 16 (1988) 351-367.

[13] Gilmour N.J.L., Gilmour J.S., Pasteurellosis of sheep, in: Adlam C., Rutter J.M. (Eds.), Pasteurella and pasteurellosis, Academic Press, London, 1989, pp. 223-262.

[14] Gilmour N.J.L., Sharp J.M., Gilmour J.S., Effect of oxytetracycline therapy on experimentally induced pneumonic pasteurellosis in lambs, Vet. Rec. 111 (1982) 97-99.

[15] Gilmour J.S., Jones G.E., Rae A.G., Quirie M., Comparison of single strains of four serotypes of Pasteurella haemolytica biotype A in experimental pneumonia of sheep, Res. Vet. Sci. 40 (1986) 136-137.

[16] Kodjo A., Villard L., Bizet C., Martel J., Sanchis R., Borges E., Gauthier D., Maurin F., Richard Y., Pulsed-Field Gel Electrophoresis is more efficient than ribotyping and random amplified polymorphic DNA analysis in discrimination of Pasteurella haemolytica strains, J. Clin. Microbiol. 37 (1999) 380-385.

[17] Mosier D.A., Confer A.W., Panciera R.J., The evolution of vaccines for bovine pneumonic pasteurellosis, Res. Vet. Sci. 47 (1989) 1-10.

[18] Mwangota A.U., Muhammed S.I., Thomson R.G., Serological types of Pasteurella haemolytica in Kenya, Cornell Vet. 68 (1978) 84-93.

[19] Mutters R., Mannheim W., Bisgaard M., Taxonomy of the group, in: Adlam C., Rutter M.J. 
(Eds.), Pasteurella and pasteurellosis, Academic Press, London, 1989, pp. 3-34.

[20] Odugbo M.O., Odama L.E., Umoh J.U., Makinde A.A., Serotypes of Pasteurella haemolytica from pneumonic lungs of sheep in northern Nigeria, Small Rumin. Res. 48 (2003) 239-243.

[21] Ojo M.O., Caprine pneumonia, Vet. Bull. 47 (1977) 573-578.

[22] Reeve-Johnson L., Relationships between clinical and pathological signs of disease in calves infected with Mannheimia (Pasteurella) haemolytica type A1, Vet. Rec. 149 (2001) 549-552.

[23] Smith G.R., Isolation of two types of Pasteurella haemolytica from sheep, Nature 183 (1959) 1132-1133.
[24] Smith G.R., The characteristics of two types of Pasteurella haemolytica associated with different pathological conditions in sheep, J. Pathol. Bacteriol. 81 (1961) 431-440.

[25] Sneath P.H.A., Stevens M., Actinobacillus rosii sp. nov., Actinobacillus seminis sp. nov., nom. rev., Pasteurella betti sp. nov., Pasteurella lymphangitidis sp. nov., Pasteurella mairi sp. nov. and Pasteurella trehalosi sp. nov., Int. J. Syst. Bacteriol. 40 (1990) 148153.

[26] Statgraphics Plus for Windows Version 2.1, http://www.statgraphics.com, Statistical Graphics Corps, 1996.

[27] Younan M., Wallmann J., A new serovar of Pasteurella haemolytica from sheep in Syria, Trop. Anim. Health Prod. 21 (1989) 87. 